\title{
Q DE CALIDAD Y SATISFACCIÓN DEL TURISTA EN EL SECTOR HOTELERO ESPAÑOL
}

\author{
M. Lilibeth Fuentes Medina ${ }^{1}$ \\ Universidad de La Laguna \\ Estefanía Hernández Estárico ${ }^{2}$ \\ Universidad Europea de Canarias \\ Sandra Morini Marrero ${ }^{1}$ \\ Universidad de La Laguna
}

\section{RESUMEN}

España ha centrado la mejora de la competitividad de sus destinos turísticos en la calidad del servicio, desarrollado a través de la marca « $Q$ de calidad turística».

En este trabajo, se contrasta si el esfuerzo realizado por los hoteles en alcanzar este estándar de calidad, es percibido por el turista que se aloja en el mismo de manera general o si se centra en alguna dimensión particular del servicio.

Los resultados revelan que, para la muestra analizada, la calidad reconocida influye en la satisfacción que obtiene el cliente en ese establecimiento de forma diferente según la dimensión objeto de estudio.

Palabras clave: Satisfacción; $Q$ de calidad; hoteles; portales de opinión.

\section{$Q$ certification and tourist satisfaction in the spanish hotels}

\section{ABSTRACT}

Spain has been working on improving the competitiveness of its tourist destinations, focusing on the quality of the service.

Recibido: 28 de mayo de 2014

Devuelto para su revisión: 7 de noviembre de 2014

Aceptado: 15 de junio de 2015

1 Departamento de Economía, Contabilidad y Finanzas. Universidad de La Laguna. Edificio de Economía, Empresa y Turismo. Campus de Guajara. Universidad de La Laguna. 38071 La Laguna. TENERIFE (España).E-mail: lfuentes@ull.edu.es,smorini@ull.edu.es

2 Departamento de Dirección de Empresas. Universidad Europea de Canarias. Calle Inocencio García, 1. 38300 La Orotava TENERIFE (España).E-mail: Estefania.hernandez@universidadeuropea.es 
This paper analyzes whether the efforts made by the tourism establishment to achieve the $Q$ brand are perceived by their guests in a general way or in a particular dimension of the service. Statistical contrasts of mean differences are performed to evaluate if the score of a $Q$ hotel is greater than that of a non-Q hotel.

The results show that the $Q$ brand is related to the satisfaction of the customer in a different way according the analyzed dimension.

Keywords: Satisfaction; $Q$-brand; hotels; online reviews.

\section{INTRODUCCIÓN}

Evaluar la satisfacción del consumidor una vez disfrutado el servicio es de vital importancia en cualquier sector, pero más aún en el ámbito del turismo, tanto a nivel de los servicios individuales (alojamiento, desplazamiento, restauración, etc.) como a nivel de destinos. La satisfacción incide en la actitud hacia el servicio y en la decisión futura de repetir o no la experiencia turística. Si el visitante se va insatisfecho, la probabilidad de repetición se reduce y no proyectará, entre sus conocidos, una imagen positiva del destino o del establecimiento (Bigné et al., 2000). Además, hay que considerar que el turista del siglo XXI tiene un mayor abanico de elección y capacidad de decisión, es un turista más exigente que pone más énfasis en la calidad que en la cantidad, y que busca sentir y experimentar vivencias (Ávila y Barrado, 2005). Así mismo, las emociones juegan un papel importante en la satisfacción y lealtad de los clientes de un hotel, de forma que, según Barsky y Nash (2003) los huéspedes estarían dispuestos a pagar más por su estancia bajo la promesa de poder experimentar ciertas emociones.

En la actualidad, el auge de las redes sociales ha incrementado el conjunto de información disponible y su accesibilidad; el número de turistas que consultan opiniones antes de reservar un hotel ha aumentado enormemente, así como el número de opiniones que se analizan antes de hacer la elección final. Desde 2010, la experiencia de otros huéspedes es el primer factor determinante en la elección de un hotel, por encima, incluso de su localización o su precio (Anderson, 2012). El aumento de las opiniones generadas por los propios usuarios del servicio y de las personas que las consultan y actúan en base a ellas, tienen un impacto económico importante que ha sido cuantificado en diferentes estudios. Así, Ye et al. (2011) concluyen que las valoraciones positivas emitidas en las redes sociales tienen un impacto directo en las ventas y Barsky y Nash (2007) cuantifican que cada comentario negativo realizado neutraliza de tres a diez comentarios positivos.

Por tanto, nunca había sido tan importante la satisfacción o insatisfacción de los usuarios, tanto por su impacto económico directo como por su influencia en las decisiones de otros potenciales clientes. Por otro lado, la importancia de la calidad en turismo también ha aumentado, en gran parte porque diversos estudios (Bolton y Drew, 1991; Danaher y Rust, 1996; Swan y Bowers, 1998; Lee et al., 2000; Soutar, 2001) han determinado que existe una relación directa y positiva entre la calidad del servicio y el nivel de satisfacción, aun cuando, como indican Parasuraman et al. (1994), otros factores como el precio también influyen en ésta. 
En definitiva, hay un creciente interés por la satisfacción del cliente y por la calidad del servicio en la medida que ésta puede generar un aumento en la satisfacción, como puede comprobarse por el crecimiento de la literatura en torno a estos factores. El binomio calidad - satisfacción ha suscitado un cambio en las políticas de desarrollo turístico en España que pasan a centrarse en el compromiso y la satisfacción del cliente. Así, ya en la década de los 90, la Dirección General de Turismo asumió que la competitividad de los destinos turísticos dependía de su imagen, organización, calidad y sostenibilidad, ya que por mucha promoción que se realizara, lo determinante era la satisfacción del turista (Foronda y García, 2009).

De esta forma, se inicia un proceso de planificación estratégica turística en el que el que la calidad del servicio para el logro de una mayor satisfacción del usuario es el eje central de la misma. Este objetivo se consolida a través del Sistema de Calidad Turística Español (SCTE) que desarrolla una metodología específica e instrumentos como la marca «Q de calidad turística» para lograr el mismo.

Considerando todo lo anterior, en este trabajo se pretende contrastar si el esfuerzo realizado por el alojamiento turístico para alcanzar el estándar de calidad español promovido a nivel institucional: $Q$ de calidad turística, es percibido por el turista que se aloja en el mismo en términos de una mayor satisfacción, medida en base a la puntuación otorgada al servicio recibido en portales online de opinión. También se desea determinar si esa diferencia de satisfacción es global o se centra en algún aspecto en particular como la limpieza del establecimiento, el trato recibido del personal u otros, habida cuenta que el proceso de certificación de la calidad hace hincapié en algunos aspectos concretos de la prestación del servicio y considerando que, como indica Stauss y Weinlich (1997), los enfoques más recientes que analizan la calidad de un servicio dividen el proceso de prestación en dimensiones claves que se evalúan de forma individual.

El trabajo está organizado tal que en el apartado 2 se introducen los conceptos y relaciones básicas de la calidad y la satisfacción, con especial referencia a los servicios de alojamiento turísticos, y al proceso de certificación de la calidad turística en España promovido desde el ámbito institucional: $Q$ de calidad turística. En el apartado 3 se muestra el impacto de los comentarios publicados en los portales de opinión. En el apartado 4 se introduce la metodología propuesta y los datos que se emplearán para contrastar la hipótesis planteada, para por último en el apartado 5 y 6 proceder a la presentación y discusión, respectivamente, de los resultados.

\section{CALIDAD Y SATISFACCIÓN EN LOS SERVICIOS DE ALOJAMIENTO}

\subsection{Marco conceptual}

Calidad y satisfacción del cliente son vistos cada vez más como parte integral de la gestión de la calidad total (Khan, 2003). Y tal y como se expuso anteriormente, como dos elementos interrelacionados (Kumar et al., 2008; Wei and Ramalu, 2011) cuyo análisis, debido a sus implicaciones económicas ${ }^{1}$, genera cada vez mayor interés. Existen diferentes

1 Se ha sugerido que la mejora de la calidad puede llevar a menores costes y márgenes de beneficio más elevados (Khan, 2003). Por otra parte, Anderson et al. (1994) concluyen que un aumento en la satisfacción del cliente tiene un impacto directo en la rentabilidad económica de la empresa. 
trabajos que tratan de establecer la relación entre ambos conceptos, pero no existe consenso sobre el sentido de la influencia. Así, algunos estudios concluyen que la satisfacción determina la calidad del servicio, otros que es la calidad la que incide en la satisfacción percibida por el usuario y un tercer grupo considera que la relación es de mutua influencia entre ambos factores ${ }^{2}$. Aunque Dabholkar (1995) señala que la relación causal depende del momento en que se evalúe el servicio. Por tanto, todavía se puede constatar la existencia de un debate relativo a la dirección de la relación entre los conceptos de calidad del servicio y satisfacción (Alén y Fraiz, 2006).

La calidad del servicio se considera un factor clave en la diferenciación de los servicios y la creación de ventajas competitivas en el turismo (Hudson et al., 2004). La calidad ha sido ampliamente estudiada en la literatura, sin embargo, no existe unanimidad acerca de su definición y mucho menos sobre el modo en que debe medirse.

Para Hudson et al. (2004) las dos herramientas principales para analizar la calidad y la satisfacción de los servicios son el IPA (Importance-performance Analysis) introducido por Martilla y James (1977) y el SERVQUAL desarrollado por Parasuraman et al. (1988). El $I P A$ es un procedimiento que se centra en la importancia relativa de determinados atributos y el desempeño del servicio bajo análisis en proporcionar tales atributos. El SERVQUAL es una escala de 22 ítems orientada a la medición de la calidad del servicio. Ambos procedimientos han evolucionado para incorporar nuevas características o adaptarse a las particularidades de servicios concretos. Así, por ejemplo, Knutson et al. (1990) proponen una alternativa al SERVQUAL específicamente para el caso de hoteles que denominan LODGSERV.

Por otro lado, es importante tener en cuenta que los clientes no perciben la calidad de un servicio como un concepto unidimensional, sino que separan la información en diferentes dimensiones (Zeithaml y Bitner, 2000). La investigación de la satisfacción en turismo comenzó en la década de los 80 , a través de la discusión de las dimensiones afectivas (Westbrook, 1987) y las dimensiones experimentales (Holbrook y Hirschman, 1982) del consumo. Estas diferentes dimensiones influyen de manera diferente en función de las variables contextuales (McCain et al., 2005; Wong y Sohal, 2003).

Existen múltiples definiciones de satisfacción ${ }^{3}$, aunque la mayoría giran en torno a un eje común relacionado con la evaluación que realiza el cliente respecto a las diferentes dimensiones del servicio recibido; evaluación que depende de la experiencia vivida y de las expectativas creadas previamente. Así, Kumar y Olshavsky (2007) diferencian entre satisfacción que es el simple cumplimiento de las expectativas y el deleite o satisfacción intensa, que supone la superación de las mismas y que conduce a sentimientos como la felicidad, la euforia o el optimismo. Según Hansemark y Albinsson (2004), «la satisfacción del cliente es una actitud general hacia un proveedor de servicios, o una reacción emocional de la diferencia entre lo que los clientes anticipan y lo que reciben, en relación con el cumplimiento de alguna necesidad, objetivo o deseo», aunque Truong y Foster (2005) no consideran que las expectativas jueguen un papel fundamental en la determinación de la satisfacción.

\footnotetext{
2 Una recopilación de estos trabajos se puede encontrar en Alén y Fraiz (2006).
}

3 Véanse, por ejemplo Gundersen et al. (1996) o Woodside et al. (1989). 
La satisfacción del cliente es la medida de cómo los clientes valoran los servicios de la organización empresarial (Gustafsson et al., 2005). Éstos perciben valor y su satisfacción se ve influida por la calidad de servicio percibida (Oh, 1999). Según Aiello et al. (2003) los clientes satisfechos son embajadores de las empresas, ya que su satisfacción puede influir a través del efecto boca-a-oreja y a la larga atraerá más clientes para la compañía.

Por tanto, la satisfacción del cliente es también una filosofía empresarial, de hecho existen prácticas empresariales que conllevan la búsqueda de la satisfacción del cliente como objetivo principal (Lin y Ding, 2005) e incluso se considera como un medio para alcanzar fines tales como ventajas competitivas, intención de compra del cliente, y en última instancia, la supervivencia (Gopalakishna y Mummalaneni, 1993).

\subsection{El sistema de calidad turística español. La marca $Q$ de calidad turística}

El actual Sistema de Calidad del Turismo Español (SCTE), sienta sus bases a principio de la década de los 90. Las empresas turísticas españolas comenzaron a percibir que las ventajas competitivas sobre las que se sustentaban sus negocios, y que habían convertido el sector turístico español en un referente tanto a nivel nacional como internacional, se estaban reduciendo considerablemente (ICTE, 2003). Para dar respuesta a esta situación, la Administración Pública Española impulsó diferentes iniciativas encaminadas a aplicar la calidad en el sector turístico como instrumento de mejora de la competitividad de los destinos turísticos españoles, desarrollándose el Sistema de Calidad de Hoteles y Apartamentos, cuyo objetivo era recuperar la ventaja competitiva de las empresas turísticas, actuando con una metodología común en todos los subsectores turísticos que estaban implicados en la mejora de la calidad de sus productos y servicios (Navarro, 1999). También se definieron una serie de estrategias basadas en la diferenciación del producto ofertado al cliente, tomando como referente la calidad del mismo. Esta primera acción pública constituyó el Plan Marco de Competitividad del Turismo Español (FUTURES I 1992-1995 y FUTURES II 1996-1999), que se desarrolló mediante el otorgamiento de subvenciones dirigidas a promover aspectos concretos como la tecnificación e innovación tecnológica de la industria turística, internacionalización de la empresa turística española, desarrollo de nuevos productos o régimen de ayudas y sistema de gestión.

Estos planes nacionales fueron sustituidos en el año 2000 y hasta el 2006, por el Plan Integral de Calidad del Turismo Español (PICTE), que profundiza en el concepto de calidad como principio inspirador que da coherencia a todas las líneas de actuación del mismo: destinos, productos, servicios sectoriales, internacionalización, cooperación internacional, formación, innovación y desarrollo tecnológico, información estadística y análisis económico, promoción y apoyo a la comercialización. Desde el año 2000, la estrategia llevada a cabo por la Administración Turística Española se tradujo en el diseño de diversos subsistemas, todos integrados en el Sistema de Calidad Turística Español (SCTE). El SCTE es un conjunto de metodologías que persigue relacionar estrechamente los conceptos de calidad, competitividad y sostenibilidad ${ }^{4}$, y se desarrolla a través de diferentes programas.

4 http://www.tourspain.es/es-es/DesarrolloSostenibilidad/Paginas/SCT.aspx 
En la actualidad y hasta el año 2020, la Administración Española trabaja con el Plan de Turismo Español Horizonte 2020, que recoge el SCTE como sistema de calidad turístico plenamente consolidado, cuyos programas de actuación evolucionaron hacia: SCTE Destinos o Sistema Integral de Calidad Turística en Destinos (SICTED), SCTE Sectores o marca $Q$ de Calidad Turística, SCTE Anfitriones y SCTE Internacional.

La $Q$ de Calidad Turística es la marca que representa la calidad en el sector turístico español y pertenece al programa SCTE Sectores, que está orientado a las Empresas y Entidades, ya que detectaron la necesidad de definir una serie de estrategias no consideradas hasta el momento y que se basaban en la diferenciación del producto ofertado al cliente, siempre tomando como referente la calidad del mismo. El Instituto para la Calidad Turística Española (ICTE) es el encargado de otorgar este distintivo que aporta a los establecimientos turísticos que la ostentan: prestigio, diferenciación, fiabilidad, rigurosidad y promoción; además de administrar y velar por el correcto uso de la marca $Q$.

Los alojamientos turísticos que deseen obtener la certificación $Q$ deben cumplir una serie de requisitos tanto relacionados con la prestación del servicio (estándares de servicio), como con los procesos internos de la empresa (estándares de gestión), y los equipamientos e infraestructuras de las instalaciones, incluido el mantenimiento y la limpieza de las mismas (estándares físicos), que se recogen en las Normas de Calidad de Servicio. Estos criterios o estándares están sometidos a un continuo proceso de cambio y adaptación, para adecuarse a la realidad del mercado y comprenden los siguientes aspectos:

(1) Estándares de gestión: se evalúa si la empresa tiene definido un sistema de calidad y si ha documentado los procedimientos a realizar. Además de la definición de los procesos se evalúa si se están aplicando las pautas establecidas en la documentación que se ha generado.

(2) Estándares de servicio: se evalúan los procesos que se llevan a cabo para prestar el servicio al cliente y si los mismos cumplen con los estándares de calidad. Tienen una puntuación asignada en función de la respuesta y en caso de no ser posible aplicar esa puntuación, se aplicarían otros sistemas de puntuación.

(3) Estándares físicos: se evalúan dos aspectos, por un lado el exterior (estética y armonía, espacio suficiente, limpieza e iluminación) y por el otro el interno, es decir, el funcionamiento de los procesos y el trabajo del día a día del personal.

El proceso de adhesión y la obtención de la certificación de la marca de calidad de las entidades turísticas, que suele implantarse en unos 9-12 meses ${ }^{5}$, sigue una serie de fases que se inicia con la solicitud de adhesión por parte del establecimiento y que culmina con la obtención de la $Q$ de calidad, una vez que se ha recibido la formación sobre la norma, ésta se ha implantado y el auditor ha dado el visto bueno acerca del proceso, que no finaliza con la concesión de la certificación, ya que se realizan periódicamente auditorías de seguimiento y renovación de la marca.

5 El tiempo de implantación puede variar en función del tamaño, la complejidad y los servicios ofrecidos. Además, la dedicación y el grado de implicación de los recursos internos implicados en el proyecto pueden aumentar o disminuir de forma significativa la duración. Otras variables que influyen son la estacionalidad y el nivel de partida de la organización. 


\section{LOS PORTALES DE OPINIÓN}

Internet ha rediseñado el modo en que la información turística se distribuye y el modo en que los turistas planifican y consumen su viaje (Buhalis y Law, 2008). Con el nacimiento de la denominada Web 2.0, los contenidos generados por los propios usuarios en las redes sociales, en las comunidades online o los portales de opinión, han pasado a ocupar el primer puesto, y en ocasiones único, del conjunto de fuentes de información que consultan otros consumidores antes de tomar una decisión (Cox et al., 2009). La influencia del boca-a-oreja, con el uso de internet, ha crecido de manera exponencial, y con ella el impacto económico directo e indirecto que tiene la opinión vertida en la red. Además, hay que considerar que las opiniones previas pueden incluso condicionar nuestras expectativas y por tanto a la satisfacción final obtenida del servicio. Es por ello, que las empresas y los propios usuarios están preocupados acerca de la credibilidad de las opiniones vertidas, en la medida que afecta a la reputación online y resultados económicos ${ }^{6}$ de las primeras y a la satisfacción de los segundos, según Díaz-Martín et al. (2000) las opiniones positivas previas hacen que las expectativas generadas sean superiores y por tanto, más difíciles de satisfacer.

Por tanto, las posibilidades que ofrece internet ha cambiado el comportamiento de los consumidores que pueden ahora interactuar directamente con los proveedores de servicios turísticos, lo que permite a estos últimos, identificar y satisfacer los cambios que requieren los usuarios de tales servicios. Y por su parte, los usuarios de los servicios turísticos reducen la incertidumbre sobre los mismos, a través de la información disponible de éstos en los foros y portales de opinión (Gursoy y McCleary, 2004).

Las opiniones publicadas en la red han sido objeto de estudio desde diferentes perspectivas. Así, Ayeh et al. (2013) examinan la credibilidad de las opiniones de TripAdvisor, que es el mayor portal de opiniones de turismo que registraba en 2012 un total de 65 millones de visitantes únicos cada mes y un total de 60 millones de opiniones. Melián-González et al. (2013) utilizando datos de TripAdvisor, concluyen que un aumento en el número de opiniones mitiga el efecto de las opiniones negativas que tienden a tomar valores desproporcionados cuando hay pocas opiniones, resultado que coincide con el obtenido por Cunningham et al. (2010) quienes concluyen que llegado un determinado número de opiniones se diluye el efecto de las opiniones extremas. Brandes et al. (2013) observan que hay un sesgo en las opiniones hacia los extremos ${ }^{7}$, de modo que, prevalecen las opiniones de los usuarios que tienen opiniones muy positivas o muy negativas que opinan de forma casi inmediata, mientras que el resto de usuarios tarda más en opinar y en ocasiones finalmente no lo hace. El sesgo tanto positivo como negativo se ha explicado habitualmente por la existencia de una propensión a opinar sólo cuando existe satisfacción (sesgo positivo) o cuando no se han cumplido las expectativas (sesgo negativo).

6 Anderson (2012) estima en $1.42 \%$ el incremento de los ingresos por habitación disponible (RevPAR), cuando aumenta la puntuación online en un punto en una escala de cinco. Y considera que se puede incrementar el precio en $11.2 \%$ con la misma probabilidad de demanda.

7 Este sesgo se detecta en estudios realizados en otros ámbitos como el de Resnik y Zeckhauser (2002) sobre Ebay o el de Chevalier y Mayzlin (2003) para opiniones online de libros. 
Se pueden distinguir distintos tipos de proveedores de información hotelera en Internet. Por un lado los que venden los servicios evaluados (páginas web de reservas/ ventas) y los que evalúan los servicios pero no los venden (páginas web de consejos sobre el alojamiento). Los primeros obtienen su beneficio porque reciben una comisión variable por habitación reservada, mientras que los segundos tienen un modelo de negocio basado en la publicidad insertada que depende de la cantidad de visitantes que tiene la página web.

En lo que respecta a los servicios de alojamiento hay dos grandes portales de opinión: TripAdvisor que como comentamos anteriormente es el portal de viajes más grande del mundo y el más estudiado desde el punto de vista académico; Booking alcanza más de 30 millones de visitantes únicos al mes y opera como intermediario en el servicio de reserva de más de 185.000 establecimientos de alojamiento turístico en 163 países.

Existen dos diferencias fundamentales entre ambos: (1) TripAdvisor es un portal exclusivamente de opinión que admite realizar reservas de manera indirecta utilizando otros portales, mientras que Booking es un portal de reserva que recopila opiniones de sus compradores y (2) las opiniones recogidas por TripAdvisor pueden ser emitidas por cualquier persona registrada en el portal aunque no necesariamente se haya alojado en el establecimiento que evalúa, mientras que en Booking sólo quienes realmente se hayan alojado pueden valorar el establecimiento y en un periodo de tiempo limitado después de haber finalizado el alojamiento.

Por tanto, el grado de fiabilidad de la información proporcionada por Booking es superior a la credibilidad de las opiniones recogidas en TripAdvisor y ha sido determinante a la hora de elegir el primero como fuente de información para nuestro estudio. En este sentido, Fernández-Barcala et al. (2010) observan la existencia de un sesgo positivo en las puntuaciones registradas en Booking respecto a TripAdvisor, que se relacionan con las diferencias de fondo existentes entre ambos portales, pero que podrían ser debidas al mayor coste de incluir críticas negativas falsas en Booking.

\section{METODOLOGÍA}

El trabajo analiza si el esfuerzo realizado por los establecimientos hoteleros para obtener la $Q$ de calidad es de algún modo percibido por el cliente, de tal forma que esto se traduce en mayores niveles de satisfacción por parte de los mismos que, a su vez, se manifiesta en unas mayores puntuaciones en los hoteles con $Q$ frente a sus homólogos (misma categoría y localización) sin $Q$. Para ello se han recopilado del portal Booking las puntuaciones globales y parciales por ítem (limpieza, confort, localización, servicio, personal y relación calidad-precio) de hoteles que disponen de la certificación $Q$ y de hoteles que no disponen de esta certificación.

Se ha elegido Booking porque al contrario que otros portales de opinión, también ampliamente reconocidos y utilizados, como Trivago o Tripadvisor, sólo pueden valorar el establecimiento quienes efectivamente han estado alojados en el mismo, solicitando el portal su opinión inmediatamente después de haber finalizado el periodo de estancia y comprende la valoración de 0 a 10 de un total de seis características o ítems sobre las cuales se calcula la puntuación promedio de cada establecimiento. Estas puntuaciones se 
consideran, en este trabajo, una proxy de la satisfacción del cliente en el establecimiento, ya que parece razonable suponer que cuanto más satisfecho se haya sentido en el establecimiento mayor será la puntuación que otorgue al mismo.

La hipótesis a contrastar es si la implantación de la $Q$ de calidad aumenta la satisfacción de los huéspedes. El análisis se realiza tanto para la puntuación global promedio como para la puntuación para cada uno de los ítems individuales disponibles y considerando tanto la muestra en su totalidad como formando diferentes submuestras que agrupen todos los hoteles de una misma categoría (tres submuestras: hoteles de 3 estrellas, 4 estrellas y 5 estrellas) o ubicados en una misma Comunidad Autónoma (diecisiete submuestras). Por tanto, se analizan en total 147 hipótesis (7 puntuaciones x 21 muestras/submuestras)

Para construir la muestra, en primer lugar se han obtenido las puntuaciones de todos los establecimientos hoteleros de tres o más estrellas que a fecha 1 de Diciembre de 2011 disponían de al menos 5 opiniones por parte de sus usuarios en Booking, resultando en un total de 4.462 establecimientos en toda España. De estos se han seleccionado todos los establecimientos que tienen reconocido el distintivo de $Q$ de calidad, obteniendo un total de 430 establecimientos cuyo detalle por categorías y localización se encuentra recogido en la Tabla 1. Por último con el fin de trabajar con una muestra equilibrada, de los 4.032 establecimientos sin Q se han seleccionado 427 establecimientos ${ }^{8}$ por muestreo aleatorio de afijación proporcional estratificado por categoría y comunidad autónoma, de forma que el número de elementos de la muestra por segmento sea proporcional al tamaño de ese segmento en el conjunto de establecimientos de ese tipo que se registran en el conjunto de datos observados; así por ejemplo, si los establecimientos ubicados en Andalucía representan aproximadamente el 17\% del total de hoteles sin Q del conjunto de datos, supondrán también un 17\% de los hoteles de la muestra: 73 (17\% de 430 establecimientos). De esta forma, la muestra está formada por un total de 857 establecimientos, 430 con $Q$ de calidad y 427 sin ella, cuya distribución por categorías y comunidades puede observarse en la Tabla 1.

Para todos ellos, además de las puntuaciones, se registra la categoría del establecimiento y su localización, que serán utilizadas como variables de control en base a los resultados obtenidos en un trabajo previo realizado por Hernández et al. (2012) quienes concluyen que las puntuaciones obtenidas por los establecimientos de diferentes categorías o situados en diferentes comunidades autónomas son significativamente distintas.

Para proceder al contraste de la hipótesis se realiza un análisis exploratorio usando intervalos de confianza para obtener una primera aproximación sobre si existen diferencias en las puntuaciones de ambos grupos de hoteles (con $Q$ y $\sin Q$ ). Si estas diferencias existen se procede a contrastar si son estadísticamente significativas a través de un contraste de medias.

Para poder elegir el contraste adecuado se necesita comprobar la normalidad y la homocedasticidad de las observaciones. Para ello se aplica el contraste de Saphiro-Francia y los contrastes de asimetría y curtosis, que determinan que las puntuaciones de cada grupo no siguen una distribución normal al rechazarse la hipótesis nula. Por su parte, el test de Levene, también indica el rechazo de la hipótesis nula de homocedasticidad de la muestra

8 La diferencia de tres establecimientos entre un grupo y otro se debe al redondeo en las proporciones. 


\section{Tabla 1 \\ NÚMERO DE ESTABLECIMIENTOS \\ EN LA MUESTRA POR GRUPO DE ESTUDIO}

\begin{tabular}{lccc}
\hline & $\begin{array}{c}\text { Con } Q \text { de } \\
\text { Calidad }\end{array}$ & $\begin{array}{c}\text { Sin } Q \text { de } \\
\text { Calidad }\end{array}$ & Total \\
\hline Global & 430 & 427 & 857 \\
\hline 3 estrellas & 120 & 220 & 340 \\
4 estrellas & 278 & 184 & 462 \\
5 estrellas & 32 & 23 & 55 \\
\hline Andalucía & 76 & 72 & 148 \\
Aragón & 13 & 16 & 29 \\
Asturias & 12 & 14 & 26 \\
C. Valenciana & 55 & 32 & 87 \\
Cantabria & 10 & 9 & 19 \\
Castilla La Mancha & 21 & 10 & 31 \\
Castilla y León & 30 & 26 & 56 \\
Cataluña & 44 & 89 & 133 \\
Extremadura & 16 & 7 & 23 \\
Galicia & 41 & 20 & 61 \\
Islas Baleares & 23 & 47 & 70 \\
Islas Canarias & 30 & 25 & 55 \\
La Rioja & 4 & 3 & 7 \\
Madrid & 8 & 36 & 44 \\
Murcia & 14 & 6 & 20 \\
Navarra & 10 & 5 & 15 \\
País Vasco & 23 & & 33 \\
\hline
\end{tabular}

Fuente: elaboración propia.

por lo que procede aplicar contrastes no paramétricos. En concreto, la prueba univariante no paramétrica para dos muestras independientes U Mann - Whitney que contrasta la hipótesis de homogeneidad, es decir, que no existen diferencias significativas entre las puntuaciones de los hoteles con $Q$ de calidad y sin ella, atendiendo a las características analizadas.

La prueba U de Mann - Whitney trabaja con rangos y comprueba lo superior que es la suma de los rangos de un subconjunto de la muestra con respecto a la suma de los rangos del otro (Novales, 1996: 449). La comparación entre la suma de rangos observadas respecto a la esperada de cada grupo determinará en cuál de ellos la puntuación analizada toma un valor significativamente mayor desde el punto de vista estadístico al del otro grupo. Así, pues el rechazo de la hipótesis nula $\left(\mathrm{H}_{0}\right)$ indicaría la existencia de diferencias, y la comparación de suma de rangos qué grupo presenta una puntuación mayor. 


\section{RESULTADOS}

Tal y como se indicó en el epígrafe anterior, en primer lugar se ha realizado un análisis exploratorio de los datos con el fin de organizarlos y detectar posibles relaciones o comportamientos entre las variables, para ello se realiza un análisis gráfico de los intervalos de confianza al $95 \%$ de la puntuación media global y por ítems de los hoteles de la muestra, tanto en su conjunto como para las diferentes submuestras consideradas (Gráficos 1 a 3$)^{9}$.

A partir de la información proporcionada por el Gráfico 1 podemos observar (líneas verticales discontinuas y panel «Promedio») que existen diferencias entre la puntuación media general que presentan los hoteles con $Q$ y los que no la tienen, siendo menor en estos últimos. A través del análisis posterior determinaremos si estas diferencias son estadísticamente significativas o no.

\section{Gráfico 1 \\ INTERVALOS DE CONFIANZA AL 95\% PARA LA PUNTUACIÓN MEDIA DE CADA ÍTEM Y LOS DOS GRUPOS CONSIDERADOS}

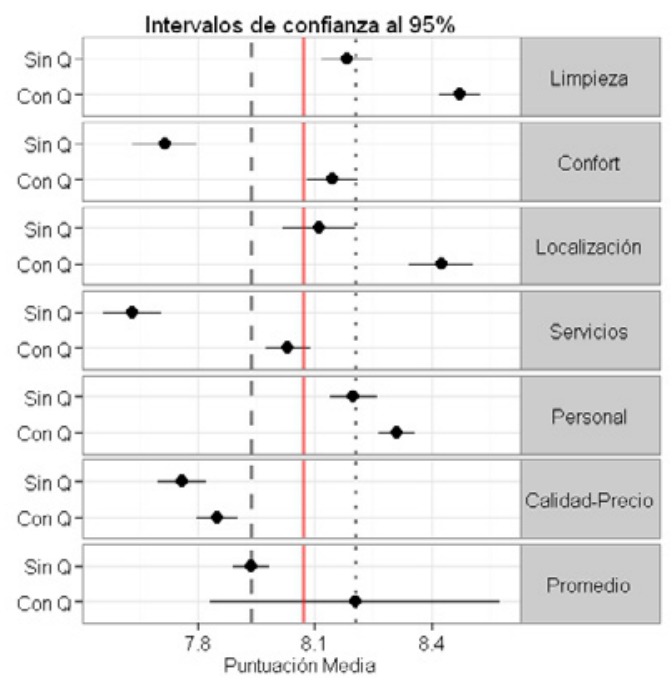

---- Promedio global hoteles $\sin Q$ — Promedio global muestra ….. Promedio global hoteles con $Q$

Para todos los ítems analizados, salvo personal y relación calidad-precio, se observan diferencias considerables entre ambos grupos de hoteles (con y $\sin Q$ ) ya que los intervalos de confianza no se solapan. Y si analizamos la posición de los intervalos de confianza de cada ítem con respecto a las medias general y por grupos, representadas verticalmente, podemos valorar la importancia de cada uno en la generación de la satisfacción promedio.

9 La línea continua vertical se muestra como referencia y representa la media de la puntuación a la que se refiere la gráfica para el conjunto de hoteles de la muestra. 
Así, limpieza, localización y personal, tienen su intervalo de confianza en valores superiores a las medias de su grupo, siendo por tanto los que tienen una valoración relativa más positiva. Destaca también que la relación calidad-precio es el ítem peor valorado en los hoteles con $Q$ y su intervalo de confianza se encuentra incluso por debajo de la puntuación media global de los hoteles $\sin Q$.

Si se efectúa el mismo análisis segmentando por categorías (Gráfico 2), se anticipa que, en general, existen diferencias entre los dos grupos de hoteles para la categoría de tres y cuatro estrellas. Caben destacar los siguientes resultados: (1) en los hoteles de tres estrellas sin distintivo $Q$ se observa una gran dispersión de la puntuación media, por tanto, desde el punto de vista del cliente que desea alojarse en un hotel de esta categoría, los hoteles con $Q$ minimizan el riesgo de obtener una experiencia poco satisfactoria, al ser más homogéneos entre sí. (2) La puntuación media global de los grupos (círculos centrales de cada intervalo) va aumentando a medida que aumenta la categoría, este comportamiento se mantiene en las puntuaciones para servicio y confort, por tanto, parece existir una relación directa entre el aumento de la categoría del hotel y la mejora en estos dos factores. Por tanto, un cliente que desee aumentar per se la calidad del servicio recibido y el confort, debería alojarse en hoteles de categoría superior; si el hotel es de cinco estrellas el hecho que tenga o no $Q$ es irrelevante, no así en los hoteles de tres o cuatro estrellas donde la $Q$ marca diferencias y permite obtener mayor satisfacción en ambos aspectos. (3) La puntuación media de limpieza y relación calidad-precio de los hoteles de tres estrellas con $Q$ es equivalente a la de cuatro estrellas $\sin Q$, en términos de puntuación para el ítem de personal los hoteles con $Q$ de tres estrellas superan a los de cuatro sin ella, por tanto, desde el punto de vista del cliente elegir un hotel de tres estrellas con certificación de calidad puede ser una opción más económica e igual o más satisfactoria en algunos ítems que alojarse en un hotel de cuatro estrellas pero sin certificación $Q$. (4) la puntuación de los hoteles de cinco estrellas es considerablemente superior a la de categorías inferiores en términos de limpieza, confort, servicios y personal, ya que no se detectan solapamientos en los intervalos de confianza de esta categoría con los de las categorías inferiores y (5) para la relación calidad-precio no parecen existir diferencias en base a la categoría del establecimiento, lo que puede indicar que la mayor calidad que se le presupone al establecimiento con la certificación $Q$ se traduce en un mayor precio, que hace que la relación entre éste y la calidad se mantenga equilibrada.

En relación a la segmentación por Comunidades Autónomas (Gráfico 3) es destacable: (1) respecto a la puntuación media general se observan diferencias en los intervalos de confianza de ambos grupos (con y $\sin Q$ ) en Andalucía, Castilla y León, Cataluña, Islas Baleares y Madrid, por tanto en estas Comunidades Autónomas, la inversión en la obtención de la $Q$ de calidad se ve compensada al generar mayor satisfacción en el cliente y por extensión, en base a lo señalado en apartados anteriores, mejorar los resultados de la empresa. (2) Atendiendo al estudio individualizado por ítems las Comunidades Autónomas donde parecen haber diferencias importantes en la puntuación media de un mayor número de ítems son: Andalucía, Cataluña, Islas Canarias y Madrid. (3) Al igual que para el estudio por categorías hoteleras no se observan diferencias entre los grupos para la puntuación media de la relación calidad-precio. 


\section{Gráfico 2}

INTERVALOS DE CONFIANZA AL 95\% DE LAS PUNTUACIONES MEDIAS SEGÚN CATEGORÍA DEL ESTABLECIMIENTO
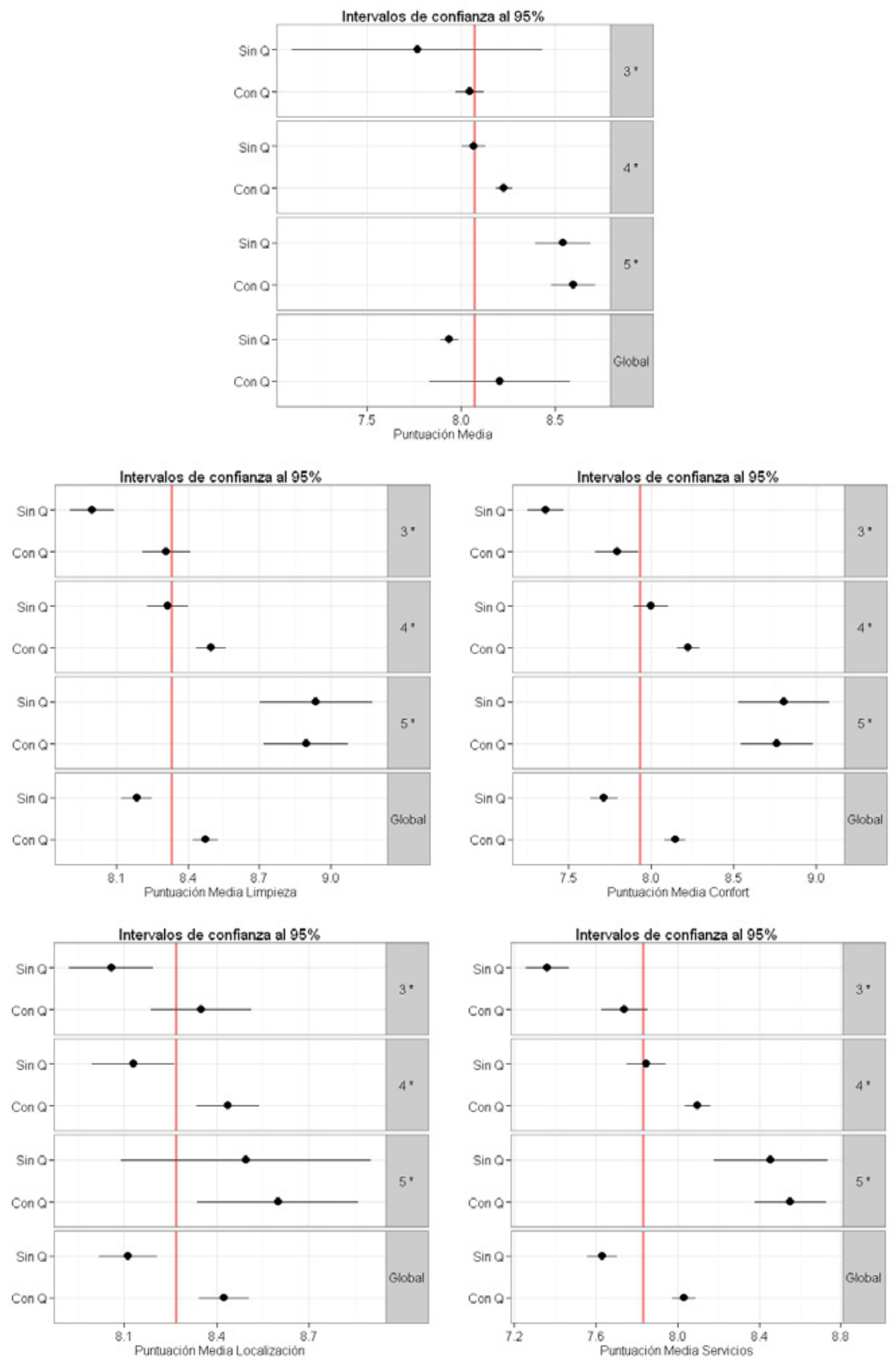

Cuadernos de Turismo, 37, (2016), 203-226 
Gráfico 2 (Continuación)

\section{INTERVALOS DE CONFIANZA AL 95\% DE LAS PUNTUACIONES MEDIAS SEGÚN CATEGORÍA DEL ESTABLECIMIENTO}
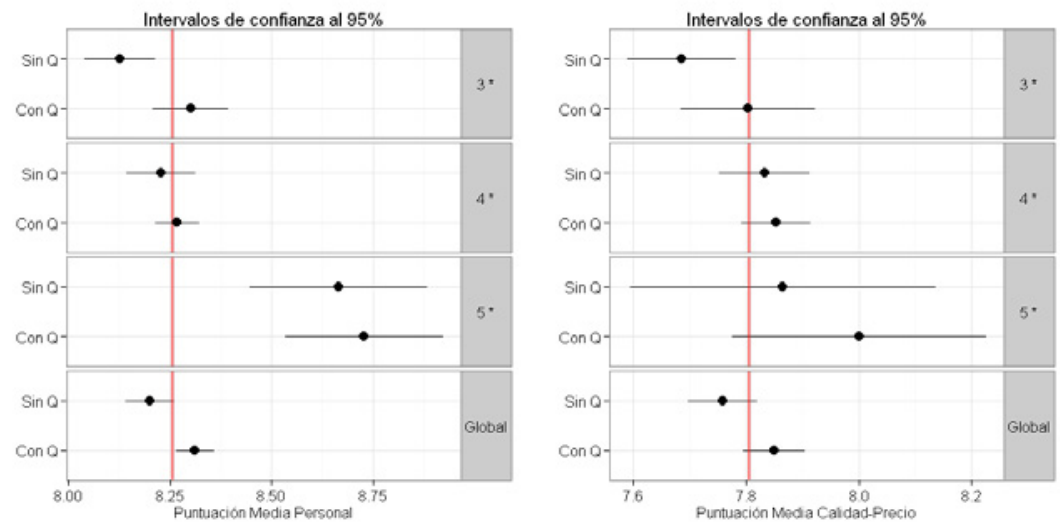

En resumen, el análisis exploratorio de los intervalos de confianza de las puntuaciones globales y por ítem de las muestras y submuestras consideradas, revelan que pueden existir en algunos casos diferencias en las puntuaciones otorgadas a los hoteles con $Q$ de calidad respecto a los que no la tienen; diferencias que tienen que ser contrastadas para determinar si son significativas o no. En caso de resultar significativas, la información proporcionada por este análisis es de gran valor para los gestores de los establecimientos, al quedar determinados por categoría y ubicación qué aspectos realmente percibe el cliente cuando el establecimiento cuenta con la certificación $Q$ de calidad.

\section{Gráfico 3 \\ INTERVALOS DE CONFIANZA AL 95\% DE LAS PUNTUACIONES MEDIAS SEGÚN COMUNIDAD AUTÓNOMA DE UBICACIÓN DEL ESTABLECIMIENTO}

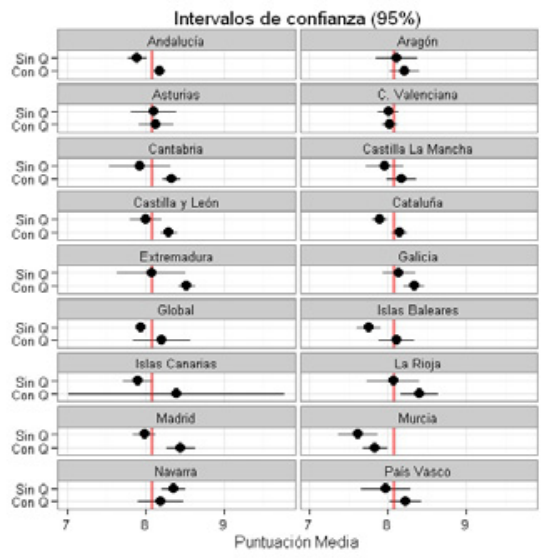




\section{Gráfico 3 (Continuación) \\ INTERVALOS DE CONFIANZA AL 95\% DE LAS PUNTUACIONES MEDIAS SEGÚN COMUNIDAD AUTÓNOMA DE UBICACIÓN DEL ESTABLECIMIENTO}
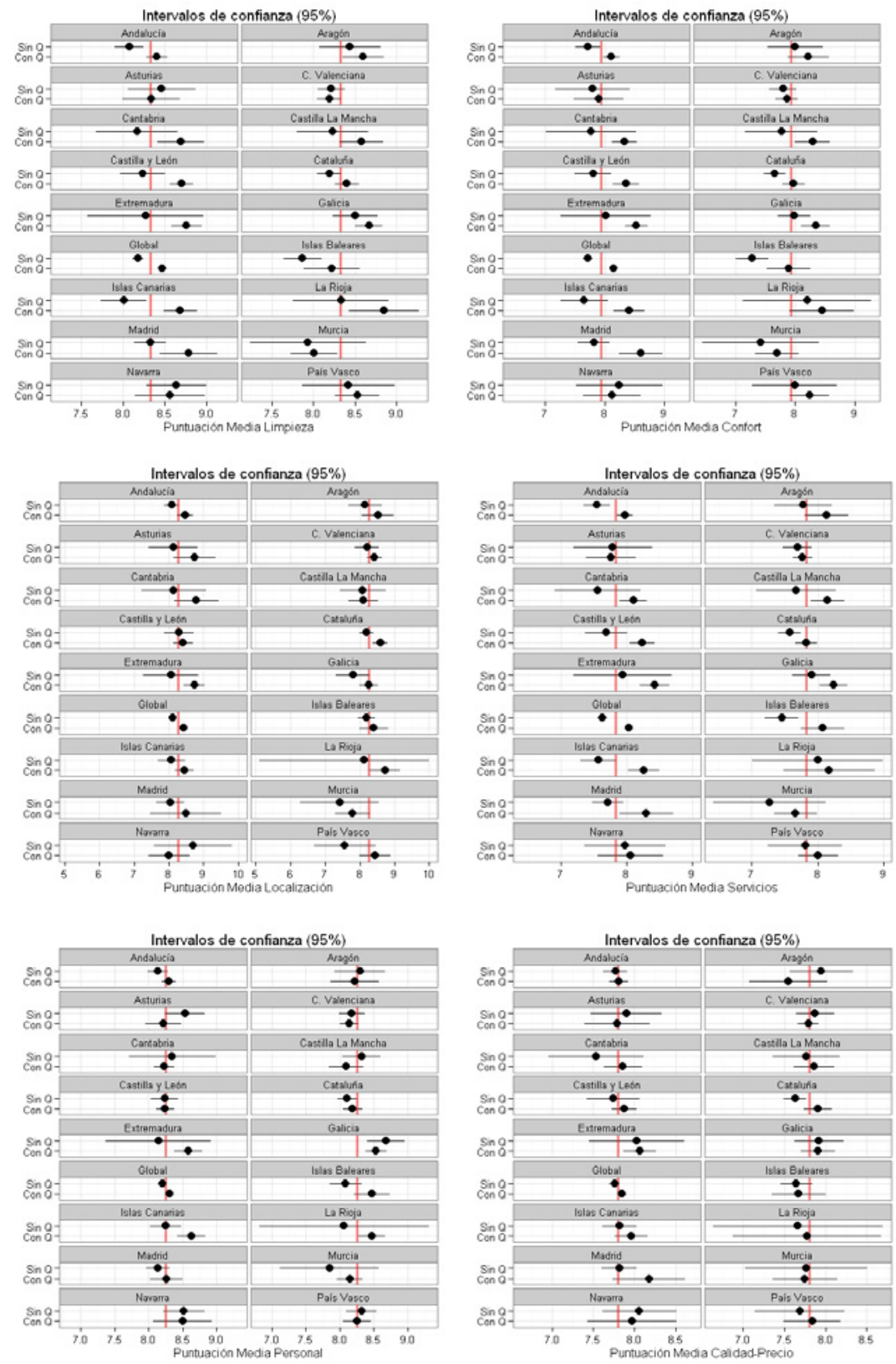
En las Tablas 2 a 7 se recogen los resultados de la aplicación del test U Mann-Whitney, sólo para las submuestras donde se ha rechazado la hipótesis nula, junto con otra información de interés. Así en cada tabla se señala el valor de la diferencia de medias entre ambos grupos de hoteles (con $Q$ y sin ella), el nivel de confianza con el que se rechaza la hipótesis nula del test U Mann - Whitney y la probabilidad de que un establecimiento con $Q$ de calidad obtenga una puntuación mayor que otro que no tiene esa distinción. Cabe destacar que tal y como adelantaban los resultados del análisis exploratorio, no se detectan diferencias entre ambos grupos de hoteles para el caso de la puntuación referente a la relación calidad-precio.

Tabla 2

\section{RESUMEN DE RESULTADOS \\ DEL ANÁLISIS DE LA PUNTUACIÓN MEDIA GLOBAL}

\begin{tabular}{lccc}
\hline & $\begin{array}{c}\text { Diferencia media } \\
\text { entre grupos }\end{array}$ & $\begin{array}{c}\text { Test U Mann-Whitney } \\
(\text { Rechazo H0) }\end{array}$ & Probabilidad \\
\hline Global & 0.267 & $* * *$ & $73.70 \%$ \\
\hline 3 estrellas & 0.279 & $* * *$ & $73.90 \%$ \\
4 estrellas & 0.162 & $* * *$ & $60.10 \%$ \\
\hline Andalucía & 0.282 & $* * *$ & $64.50 \%$ \\
Cataluña & 0.261 & $* * *$ & $64.80 \%$ \\
Extremadura & 0.454 & $* *$ & $77.20 \%$ \\
Islas Baleares & 0.358 & $* *$ & $66.10 \%$ \\
Islas Canarias & 0.493 & $* * *$ & $73.60 \%$ \\
Madrid & 0.467 & $* * *$ & $79.90 \%$ \\
\hline$* *$ 99\% de confianza & $* * 95 \%$ de confianza & &
\end{tabular}

De los resultados obtenidos cabe destacar que, tanto para la puntuación media general como para la de todos los ítems analizados salvo la relación calidad-precio, se observan diferencias estadísticamente significativas para toda la muestra y para las submuestras de hoteles de tres estrellas y la submuestra de hoteles de Andalucía.

Para la muestra en su conjunto, las mayores diferencias entre ambos tipos de establecimientos se registran en confort y servicios y la menor en personal. Así mismo se estima en un $73.7 \%$ la probabilidad de que un hotel con $Q$ obtenga una puntuación mayor que otro que no la tiene. Probabilidad que alcanza casi un $80 \%$ para los establecimientos de la Comunidad Autónoma de Madrid.

Si nos centramos en las categorías hoteleras, la obtención de la $Q$ de calidad es valorada positivamente en los establecimientos de 3 y 4 estrellas pero no hay evidencias de que mejore la puntuación en los de la categoría superior. En los hoteles de 3 estrellas las diferencias promedio mayores se registran en confort y servicios y la menor en personal, en los hoteles de 4 estrellas el ítem más relevante es la localización. 
Tabla 3

RESUMEN DE RESULTADOS DEL ANÁLISIS

DE LA PUNTUACIÓN MEDIA DE LIMPIEZA

\begin{tabular}{lccc}
\hline & $\begin{array}{c}\text { Diferencia media } \\
\text { entre grupos }\end{array}$ & $\begin{array}{c}\text { Test Mann-Whitney } \\
\left(\text { Rechazo } \mathrm{H}_{0}\right)\end{array}$ & Probabilidad \\
\hline Global & 0.289 & $* * *$ & $62.90 \%$ \\
\hline 3 estrellas & 0.313 & $* * *$ & $63.00 \%$ \\
\hline 4 estrellas & 0.180 & $* * *$ & $60.10 \%$ \\
\hline Andalucía & 0.330 & $* * *$ & $65.10 \%$ \\
\hline Castilla y León & 0.462 & $* *$ & $69.70 \%$ \\
\hline Islas Canarias & 0.679 & $* * *$ & $80.40 \%$ \\
\hline La Rioja & 0.517 & $* *$ & $95.80 \%$ \\
\hline Madrid & 0.463 & $* *$ & $78.10 \%$ \\
\hline$* * * 99 \%$ de confianza & $* * 95 \%$ de confianza & &
\end{tabular}

Tabla 4

RESUMEN DE RESULTADOS DEL ANÁLISIS

DE LA PUNTUACIÓN MEDIA DE CONFORT

\begin{tabular}{lccc}
\hline & $\begin{array}{c}\text { Diferencia media } \\
\text { entre grupos }\end{array}$ & $\begin{array}{c}\text { Test Mann-Whitney } \\
\left(\text { Rechazo } \mathrm{H}_{0}\right)\end{array}$ & Probabilidad \\
\hline Global & 0.431 & $* * *$ & $65.00 \%$ \\
\hline 3 estrellas & 0.432 & $* * *$ & $64.90 \%$ \\
\hline 4 estrellas & 0.227 & $* * *$ & $59.70 \%$ \\
\hline Andalucía & 0.398 & $* * *$ & $64.30 \%$ \\
\hline Cantabria & 0.563 & $* *$ & $75.00 \%$ \\
\hline Castilla y León & 0.553 & $* * *$ & $72.40 \%$ \\
\hline Cataluña & 0.319 & $* *$ & $61.60 \%$ \\
\hline Galicia & 0.366 & $* *$ & $70.40 \%$ \\
\hline Islas Baleares & 0.615 & $* *$ & $68.60 \%$ \\
\hline Islas Canarias & 0.755 & $* * *$ & $72.10 \%$ \\
\hline Madrid & 0.786 & & $85.10 \%$ \\
\hline
\end{tabular}

*** $99 \%$ de confianza $\quad * * 95 \%$ de confianza 


\section{Tabla 5 \\ RESUMEN DE RESULTADOS DEL ANÁLISIS DE LA PUNTUACIÓN MEDIA DE LOCALIZACIÓN}

\begin{tabular}{lccc}
\hline & $\begin{array}{c}\text { Diferencia media } \\
\text { entre grupos }\end{array}$ & $\begin{array}{c}\text { Test Mann-Whitney } \\
\left(\text { Rechazo } \mathrm{H}_{0}\right)\end{array}$ & Probabilidad \\
\hline Global & 0.313 & $* * *$ & $59.10 \%$ \\
3 estrellas & 0.292 & $* *$ & $58.00 \%$ \\
4 estrellas & 0.309 & $* * *$ & $59.70 \%$ \\
Andalucía & 0.398 & $* * *$ & $62.80 \%$ \\
Cataluña & 0.399 & $* *$ & $62.60 \%$ \\
\hline
\end{tabular}

*** 99\% de confianza $\quad * * 95 \%$ de confianza

En cuanto al estudio por Comunidades Autónomas, para la puntuación media general, la diferencia entre ambos grupos de hoteles es mayor en Canarias, Madrid y Extremadura donde alcanza casi medio punto. En la puntuación de limpieza, destaca una gran diferencia entre grupos en Canarias y La Rioja por encima de 0.5. En lo que respecta al confort, los hoteles con $Q$ de Madrid y Canarias superan en media casi 0.8 puntos a los que no la tienen.

\section{Tabla 6 \\ RESUMEN DE RESULTADOS DEL ANÁLISIS DE LA PUNTUACIÓN MEDIA DE SERVICIOS}

\begin{tabular}{lccc}
\hline & $\begin{array}{c}\text { Diferencia media } \\
\text { entre grupos }\end{array}$ & $\begin{array}{c}\text { Test Mann-Whitney } \\
\left(\text { Rechazo } \mathrm{H}_{0}\right)\end{array}$ & Probabilidad \\
\hline Global & 0.401 & $* * *$ & $65.60 \%$ \\
\hline 3 estrellas & 0.376 & $* * *$ & $64.20 \%$ \\
\hline 4 estrellas & 0.251 & $* * *$ & $61.60 \%$ \\
\hline Andalucía & 0.424 & $* * *$ & $67.60 \%$ \\
\hline Castilla y León & 0.544 & $* * *$ & $71.40 \%$ \\
\hline Cataluña & 0.259 & $* *$ & $62.10 \%$ \\
\hline Galicia & 0.339 & $* *$ & $67.90 \%$ \\
\hline Islas Baleares & 0.625 & $* *$ & $68.90 \%$ \\
\hline Islas Canarias & 0.688 & $* * *$ & $78.10 \%$ \\
\hline Madrid & 0.589 & $* * *$ & $81.10 \%$ \\
\hline$* * *$ 99\% de confianza & $* * 95 \%$ de confianza & &
\end{tabular}

Por último la localización y el personal no son ítems que generen diferencias importantes salvo en casos puntuales y con una probabilidad relativamente baja. 
En definitiva, los contrastes de hipótesis realizados, confirman los resultados que se adelantaban en el análisis exploratorio y permiten reforzar las conclusiones y comentarios efectuados previamente.

\section{Tabla 7 \\ RESUMEN DE RESULTADOS DEL ANÁLISIS \\ DE LA PUNTUACIÓN MEDIA DE PERSONAL}

\begin{tabular}{lccc}
\hline & $\begin{array}{c}\text { Diferencia media } \\
\text { entre grupos }\end{array}$ & $\begin{array}{c}\text { Test Mann-Whitney } \\
\left(\text { Rechazo } \mathrm{H}_{0}\right)\end{array}$ & Probabilidad \\
\hline Global & 0.112 & $* * *$ & $55.50 \%$ \\
\hline 3 estrellas & 0.174 & $* * *$ & $58.50 \%$ \\
\hline Andalucía & 0.157 & $* *$ & $60.60 \%$ \\
\hline Islas Canarias & 0.382 & $* *$ & $70.20 \%$ \\
\hline$* *$ 99\% de confianza & $* * 95 \%$ de confianza & &
\end{tabular}

\section{DISCUSIÓN DE RESULTADOS}

Los resultados parecen confirmar que, en general, el esfuerzo que realizan los establecimientos hoteleros españoles por alcanzar el estándar de calidad turística ( $Q$ de calidad turística) es percibido por los clientes, a través de la mejor puntuación que éstos otorgan una vez disfrutada la experiencia del servicio; si bien, el impacto que tiene la certificación de la calidad no es homogéneo y depende de la categoría del establecimiento y su localización; los directivos de los hoteles tienen en este resultado una fuente de motivación más allá de los factores internos que parecen primar a la hora de decidir la adhesión al proceso de certificación, según indican Tarí et al. (2012).

En los hoteles de cinco estrellas las diferencias entre ambos grupos de hoteles (con y sin certificación $Q$ ) no es significativa. Esto puede deberse a que en esta categoría la calidad media de los servicios ya de por sí es elevada y el cliente no observa realmente una diferencia significativa entre unos hoteles y otros. La diferencia es mayor en los hoteles de tres estrellas, por lo que, en éstos, sí que invertir en la obtención del certificado puede ser rentable. Por tanto, los hoteles de tres estrellas son en los que más se hace patente la mejora derivada del proceso de certificación, llegando en algunos ítems a ofrecer una satisfacción igual o superior que un establecimiento de cuatro estrellas pero $\sin Q$ de calidad. En los hoteles de cuatro estrellas, también se registran mejoras pero no son lo suficientemente potentes como para que puedan equipararse a los hoteles de categoría superior.

Así mismo, el efecto positivo de la certificación no se observa en todas las Comunidades Autónomas; por ejemplo, en Canarias, Madrid o Extremadura, la diferencia entre ambos grupos de hoteles es casi de medio punto. Atendiendo a los resultados de Anderson (2012) esto se traduciría en que un hotel con $Q$ tendría un mayor ingreso por habitación disponible y podría facturar el servicio a un mayor precio que otro hotel $\sin Q$ de características homogéneas. 
Las implicaciones prácticas de los resultados son directas. Los directivos hoteleros son responsables de la satisfacción de sus clientes, pero a menudo tienen poca orientación sobre cómo determinar si la experiencia del servicio ha sido satisfactoria (Yuksel y Rimmington, 1998). Por otra parte, mejorar la calidad requiere identificar las causas de las deficiencias y desarrollar estrategias para minimizarlas (Alén y Rodríguez, 2004). En este trabajo, se identifica que los procesos de certificación en calidad para la obtención del distintivo $Q$ de calidad turística, parecen ser más patentes en el ámbito de la prestación de los servicios del hotel y el confort de sus huéspedes, que son los ítems donde la diferencia de puntuación entre ambos grupos es mayor. Por tanto, cabe suponer que la obtención de la certificación $Q$ redunda más directamente en estos dos aspectos, aunque también se detecten diferencias en el ámbito de la limpieza o el personal del hotel, que son ambos ítems con una puntuación por encima de la media global.

En este sentido, cabe destacar que Choi y Chu (2001) concluyen que la infraestructura del hotel, la calidad de sus habitaciones y servicio (que podemos identificar con el atributo de confort analizado en este trabajo) y el personal son factores claves que afectan a la satisfacción del cliente. De igual forma, Qu et al. (2000) para su muestra de establecimientos hoteleros concluyen que el personal es el factor más importante, seguido del equipamiento, la relación calidad - precio y los servicios. En concreto, analizando la $Q$ de calidad turística, Álvarez et al. (2013) concluyen que el factor humano es el más importante para lograr el éxito en la implantación del sistema de calidad.

En consecuencia, los ítems utilizados para realizar el análisis son relevantes y proporcionan conclusiones valiosas ya que son los que más relacionados están con la satisfacción del cliente. Por tanto, se puede concluir que a la luz de los resultados obtenidos, en términos globales, los clientes que se alojan en establecimientos que tienen la certificación $Q$ se sienten más satisfechos; aunque esta mayor satisfacción ni en algunas Comunidades Autónomas y ni para la categoría de cinco estrellas es significativa.

La contribución de este trabajo a la literatura, es que amplía los resultados de estudios previos, como el de Tarí et al. (2012), Álvarez et al. (2013) o del Rio et al. (2013), analizando un sistema de calidad específico (la $Q$ de calidad turística); ya que aporta la perspectiva del cliente (turista) y su percepción (medida en términos de satisfacción) sobre la eficiencia del sistema de calidad, a través de una mejor valoración de la experiencia del servicio. Sin embargo, como sugieren Alén y Rodríguez (2004) la forma en la que los clientes evalúan la calidad del servicio no sólo depende del servicio que se está evaluando sino que incluso puede depender del segmento analizado, es por ello que la consideración de la segmentación de la demanda en el análisis, puede ser una interesante línea de investigación futura.

\section{BIBLIOGRAFÍA}

AIELLO, A., GARMAN, A. y MORRIS, B.S. (2003): «Patient satisfaction with nursing care: A multilevel analysis». Journal of Quality Management in Health Care, $\mathrm{n}^{\circ} 12$ (3), pp. 187-191.

ALÉN, M E. y FRAIZ, J.A. (2006): «Relación entre la calidad de servicio y la satisfacción del consumidor. Su evaluación en el ámbito del turismo termal». Investigaciones Europeas de Dirección y Economía de la Empresa, nº 12(1), pp. 251-272. 
ALÉN, M.E. y RODRÍGUEZ, L. (2004): «Evaluación de la calidad percibida por los clientes de establecimientos termales a través del análisis de sus expectativas y percepciones». Revista Galega de Economía, n 14(1-2), pp. 1-18.

ÁLVAREZ, J., FRAIZ, J.A. y DEL RIO, M.C. (2013): «Q de calidad turística. Barreras a la implantación y certificación». Cuadernos de Turismo, no 32, pp. 11-35.

ANDERSON, C. (2012): The impact of social media on lodging performance. Cornell Hospitality Report, Noviembre.

ANDERSON, E., FORNELL, C. y LEHMANN, D. (1994): «Customer satisfaction, market share and profitability: findings from Sweden». Journal of Marketing, $\mathrm{n}^{\circ}$ 58, pp. 53-66.

ÁVILA, R. y BARRADO, D.A. (2005): «Nuevas tendencias en el desarrollo de destinos turísticos: marcos conceptuales y operativos para su planificación y gestión». Cuadernos de Turismo, $\mathrm{n}^{\circ} 15$, pp. 27-43.

AYEH, J. K, AU, N. y LAW, R. (2013): «Do We Believe in TripAdvisor? Examining Credibility Perceptions and Online Travelers> Attitude toward Using User-Generated Content». Journal of Travel Research, $\mathrm{n}^{\circ}$ 52(4), pp. 437-452.

BARSKY, J. y NASH, L. (2003): «Customer Satisfaction». Cornell Hotel and Restaurant Administration Quarterly, pp. 173-183.

BARSKY, J. y NASH, L. (2007): «Word of Mouth should be top of mind for hotel operators», Hotel \& Motel Management. Market Metrix.

BIGNÉ, E., FONT, X. y ANDREU, L. (2000): Marketing de Destinos Turísticos. Análisis y Estrategias de desarrollo, ESIC, Madrid.

BOLTON, R. N. y DREW, J. H. (1991): «A Multistage Model of Customers Assessments of Service Quality and Value». Journal of Consumer Research, $\mathrm{n}^{\circ}$ 17(4), pp. 375-384.

BRANDES, L., GODES, D., y MAYZLIN, D. (2013): Controlling for Self-Selection Bias in Customer Reviews. Manuscrito. Disponible en: https://msbfile03.usc.edu/ digitalmeasures/mayzlin/intellcont/SelfSelectionOnlineReviews-1.pdf

BUHALIS, D. y LAW, R. (2008): «Progress in information technology and tourism management: 20 years on and 10 years after the Internet-the state of eTourism research». Tourism Management, $\mathrm{n}^{\circ} 29$ (4), pp. 609-623.

CHEVALIER, J.A. y MAYZLIN, D. (2003): The effect of word of mouth on sales: Online book reviews. NBER working paper, 10148.

CHOI, T.Y. y CHU, R. (2001): «Determinants of hotel guests' satisfaction and repeat patronage in Hong Kong hotel industry». International Journal of Hospitality Management, $\mathrm{n}^{\circ} 20$, pp. 277-297.

COX, C., BURGESS, S., SELliTTO, C. y BUULTJENS, J. (2009): «The Role of UserGenerated Content in Tourists' Travel Planning Behavior». Journal of Hospitality Marketing and Management, $\mathrm{n}^{\circ}$ 18, pp. 743-64.

CUNNINGHAM, P., SMYTH, B., WU, G. y GREENE, D. (2010): Does TripAdvisor makes hotels better?. Technical Report UCD-CSI-2010-6.

DABHOLKAR, P.A. (1995): «The convergence of customer satisfaction and service quality evaluations with increasing customer patronage». Journal of Consumer Satisfaction, Dissatisfaction and Complaining Behavior, $\mathrm{n}^{\circ}$ 8, pp. 32-43.

DANAHER, P.J. y RUST, R.T. (1996): «Indirect Marketing Benefits From Service Quality». Quality Management Journal, n 3(2), pp. 63-88. 
DEL RIO, M.C., ÁLVAREZ, J. y VILA, M. (2013): «Reasons which lead the rural accommodation sector to become certified with «Q for Tourist Quality»». Global Business Perspectives, $\mathrm{n}^{\mathrm{o}}$ 1, pp 341-358.

DIAZ-MARTIN, A.M., IGLESIAS, V., VAZQUEZ, R. y RUIZ, A.V. (2000): «The use of quality expectations to segment a service market». Journal of Services Marketing, $\mathrm{n}^{\mathrm{o}}$ 14 (2), pp. 132-146.

FERNÁNDEZ-BARCALA, M., GONZÁLEZ-DÍAZ, M., y PRIETO-RODRÍGUEZ, J. (2010): «Hotel quality appraisal on the Internet: a market for lemons?». Tourism Economics, $\mathrm{n}^{\circ} 16(2)$, pp. 345-360.

FORONDA, C. y GARCÍA, A.M. (2009): «La apuesta por la calidad como elemento diferenciador en los destinos turísticos: planes renovados». Cuadernos de Turismo, $\mathrm{n}^{\circ} 23$, pp. 89-11.

GOPALAKISHNA, P. y MUMMALANENI, V. (1993): «Influencing satisfaction for dental services». Journal of Health Care Market, $\mathrm{n}^{\circ}$ 13(1), p. 16-22.

GUNDERSEN, M.G., HEIDE, M. y OLSSON, U.H. (1996): «Hotel Guest Satisfaction among Business Travelers. What are the important factors?». Cornell hotel and restaurant administration quarterly, $\mathrm{n}^{\circ}$ abril, pp. 72-81.

GURSOY, D. y MCCLEARY, K. (2004): «An integrative model of tourism information search behavior». Annals of Tourism Research, $\mathrm{n}^{\circ} 31(2)$, pp. 353-373.

GUSTAFSSON, A., JOHNSON, M.D. y ROOS, I. (2005): «The Effects of Customer Satisfaction, Relationship Commitment Dimensions, and Triggers on Customer Retention». Journal of Marketing, $\mathrm{n}^{\circ}$ 69, pp. 210-218.

HANSEMARK, O.C. y ALBINSON, M. (2004): «Customer Satisfaction and Retention: The Experiences of Individual Employees». Managing Service Quality, n 14 (1), pp. 40-57.

HERNÁNDEZ-ESTÁRICO, E., FUENTES-MEDINA, M.L. y MORINI-MARRERO, S. (2012): «Una aproximación a la reputación en línea de los establecimientos hoteleros españoles». Papers de Turisme, $\mathrm{n}^{\circ}$ 52, pp. 63-88.

HOLBROOK, M.B. y HIRSCHMAN, E.C. (1982): «The experiential aspects of consumption: consumer fantasies, feelings and fun». Journal of Consumer Research, $\mathrm{n}^{\mathrm{o}}$ 9, pp. 132-140.

HUDSON, S., HUDSON, P. y MILLER, G. ${ }^{\circ}$ A. (2004): «The Measurement of Service Quality in the Tour Operating Sector: A Methodological Comparison». Journal of Travel Research, $\mathrm{n}^{\mathrm{O}}$ 42, pp. 305-312.

ICTE (Instituto Calidad Turística Española) (2003): Información General SCTE. Recuperado el día 26 de Julio de 2013 desde: http://www.profesionales.calidadturistica.es/ index.aspx

KHAN, M. (2003): «ECOSERV. Ecotourists' Quality Expectations». Annals of Tourism Research, no 30 (1), p. 109-124.

KNUTSON, B., STEVENS, P., WULlAERT, C., PATTON, M. y YOKOYAMA, F. (1990): LODGSERV: «A Service Quality Index for the Lodging Industry». Journal of Hospitality \& Tourism Research, $\mathrm{n}^{\circ} 14(2)$, pp. 277-284.

KUMAR, A. y OLSHAVSKY, R. (2007): «A distinguishing satisfaction from delight: an appraisal approach». Annual Conference of the Association for Consumer Research, Tucson, Arizona. 
KUMAR, V., SMART, P.A., MADDERN, H. y MAULL, R.S. (2008): «Alternative Perspective on Service Quality and Customer Satisfaction: the Role of BPM». International Journal of Service Industry Management, $\mathrm{n}^{\circ} 19$ (2), pp. 176-187.

LEE, H., LEE, Y. y YOO, D. (2000): «The determinants of perceived service quality and its relationship with satisfaction». Journal of Service Marketing, $\mathrm{n}^{\circ} 14$ (3), pp. 217-231.

LIN, C.P. y DING, C.G. (2005): «Opening the black box: assessing the mediating mechanism of relationship quality and the moderating effects of prior experience in ISP service». International Journal of Service Industry Management, $\mathrm{n}^{\circ}$ 16(1), pp. 55-80.

MARTILLA, J.A. y JAMES, J.C. (1977): «Importance-Performance Analysis». Journal of Marketing, $\mathrm{n}^{\circ} 41$ (3), pp. 77-79.

McCAIN, S L., JANG, S. y HU, C. (2005): «Service quality gap analysis toward customer». Hospitality Management, $\mathrm{n}^{\circ}$ 24, pp.465- 472.

MELIÁN-GONZÁLEZ, S., BULCHAND-GIDUMAL, J. y GONZÁLEZ LÓPEZ-VALCÁRCEL, B. (2013): «Online customer reviews of hotels: as participation increases, better evaluation is obtained». Cornell Hospitality Quarterly, no 29, pp. 1-10.

NAVARRO, A. (1999): «Plan de calidad turística española». Estudios Turísticos, n 139 , pp. 5-13.

NOVALES, A. (1996): Estadística y Econometría. Ed. McGraw-Hill. Madrid

OH, H. (1999): «Service quality, customer satisfaction and customer value: a holistic perspective». International Journal of Hospitality Management, $\mathrm{n}^{\circ}$ 18, pp. 67-82.

PARASURAMAN, A.; ZEITHAML, V. y BERRY, L. (1988): SERVQUAL: «A MultipleItem Scale for Measuring Consumer Perceptions of Service Quality». Journal of Retailing, $\mathrm{n}^{\circ} 64$ (1), pp. 12-40.

PARASURAMAN, A., ZEITHAML, V. y BERRY, L. (1994): Reassessment of expectations as a comparison standard in measuring service quality: Implications for further research». Journal of Marketing, ${ }^{\circ}$ 58, pp. 120-135.

QU, H., RYAN, B. y CHU, R. (2000): «The Importance of Hotel Attributes in Contributing to Travelers' Satisfaction in the Hong Kong Hotel Industry». Journal of Quality Assurance in Hospitality and Tourism, n ${ }^{\circ}$ 1(3), pp. 65-83.

RESNICK, P. y ZECKHAUSER, R. (2002): «Trust among strangers in internet transactions: Empirical analysis of eBay's reputation system». Advances in applied microeconomics, $\mathrm{n}^{\mathrm{o}} 11$, pp. 127-157.

SOUTAR, J. N. (2001): «Service quality, customer satisfaction and value: An examination on their relationships». In J. Kandampuly, C. Mok, y B. Sparks (Eds.). Service quality management in hospitality, tourism and leisure. New York: The Haworth Press.

STAUSS, B. y WEINLICH, B. (1997): «Process-Oriented Measurement of Service Quality. Applying the Sequential Incident Technique». European Journal of Marketing, $\mathrm{n}^{\circ}$ 31 (1), pp. 33-35.

SWAN, J.E. y BOWERS, M.R. (1998): «Services Quality and Satisfaction: The Process of People Doing Things Together». Journal of Services Marketing, $\mathrm{n}^{\circ} 12$ (1), pp. 59-72.

TARÍ, J.J., HERAS-SAIZARBITORIA, I. y DICK, G. (2012): Internal and external drivers for quality certification in the service industry: Do they have different impacts on success?. Service Business, Disponible en: http://ssrn.com/abstract=2041039. 
TRUONG, T.H. y FOSTER, D. (2005): «Using HOLSAT to evaluate tourist satisfaction at destinations: The case of Australian holidaymakers in Vietnam». Tourism Management, $\mathrm{n}^{\mathrm{o}} 27$, pp. 842-855.

WEI, C.C. y RAMALU, S.S. (2011): «Students Satisfaction towards the University: Does Service Quality Matters». International Journal of Education, $\mathrm{n}^{\circ} 3$ (2), pp. E15.

WESTBROOK, R. A. (1987): «Product/Consumption-based Affective Responses and Postpurchase Processes». Journal of Marketing Research, $\mathrm{n}^{\circ} 24$, pp. 258-270.

WOODSIDE, A.G., FREY, L.L. y DALY, R.T. (1989): «Linking Service Quality, Customer Satisfaction, and Behavioral Intention». Journal of Health Care Marketing, $\mathrm{n}^{\circ}$ 9, pp. 5-17.

WONG, A. y SOHAL, A. (2003): «Assessing customer-salesperson interactions in a retail chain: Differences between city and country retail districts». Marketing Intelligence and Planning, $\mathrm{n}^{\circ} 21$ (5), pp. 292-304.

YE, Q., LAW, R., GU, B. y CHEN, W. (2011): «The influence of user-generated content on traveler behavior: An empirical investigation on the effects of e-word-of-mouth to hotel online bookings». Computers in Human Behavior, $\mathrm{n}^{\circ} 27$ (2), pp. 634-639.

YUKSEL, A. y RIMMINGTON, M. (1998): «Customer-Satisfaction Measurement» . Cornell Hotel and Restaurant Administration Quarterly, $\mathrm{n}^{\circ}$ 39(6), pp. 60-71.

ZEITHAML, V.A. y BITNER, M.J. (2000): Services marketing: Integrating customer focus across the firm. $2^{\text {a }}$ Ed. Boston. McGraw-Hill. 